\title{
Accountability em Sistemas de Informação
}

\author{
Felipe Cordeiro de Paula ${ }^{1,2}$, Rodrigo Pereira dos Santos ${ }^{1}$ \\ ${ }^{1}$ PPGI - Universidade Federal do Estado do Rio de Janeiro (UNIRIO) \\ Rio de Janeiro, Brasil \\ ${ }^{2}$ Instituto Superior de Educação do Rio de Janeiro (ISERJ/FAETEC) \\ Rio de Janeiro, Brasil \\ fcp.cordeiro@iserj.edu.br, rpseuniriotec.br
}

\begin{abstract}
This paper reports a PhD work about accountability and Information Systems (IS). We highlight the need for a research agenda where complex systems must value three accountability fundamentals as a way to improve sustainability in systems: engagement, management of activities and compliance. In order to do so, we propose to extend the meaning of accountability and elaborate a framework for evaluating accountability in IS.

Resumo. Este artigo relata uma pesquisa de doutorado sobre accountability e Sistemas de Informação (SI). Destacamos a necessidade de uma agenda de pesquisa em que sistemas complexos devem valorizar três fundamentos de accountability como formar de aperfeiçoar sustentabilidade em sistemas: engajamento, gerenciamento das atividades e observância a regras. Para tanto, propomos ampliar a significação de accountability e elaborar um framework de avaliação de accountability em SI.
\end{abstract}

\section{Introdução}

O conceito de accountability e suas aplicações vêm chamando atenção de diversas áreas de conhecimento. Na Administração Pública, é associada ao processo de prestar contas para alguma autoridade [Mulgan 2000]. Em Ciência da Computação (CC), tem se referido aos processos e mecanismos de autorização e segurança [Feigenbaum et al. 2012]. Em Educação, o conceito é associado a políticas educacionais para obtenção de melhores indicadores, pelo incentivo à inspeção de dados e associação à qualidade de ensino [Wolf e Janssens 2007].

Em Sistemas de Informação (SI), a comunidade discute os desafios de usuários de sistemas fortalecerem a accountability individual por meio dos estímulo a apropriação de responsabilidade e diálogo [Araujo 2016]. Van Bussel (2016) discorre sobre accountability organizacional e o desafio de obter dados reais representativos dos contextos e memória na organização. Enquanto isso, Kacianka e Pretschner (2018) propõem um modelo formal para destacar os mecanismos em sistemas, identificando suas capacidades e expressando diferentes noções de accountability no contexto de Cyberphysical Systems (CPS) ${ }^{1}$.

Contudo, cabe destacar que, apesar desta diversidade de áreas de conhecimento, não há uma definição formal de accountability universalmente aceita. Diversos artigos científicos utilizam o termo de forma vaga, onde não são apresentados significados ou não são detalhados os recursos accountable presentes nas soluções [Medeiros et al. 2013].

Assim, para disciplinar um conceito, neste trabalho utilizamos a definição do IS.Theorizeit.org, em que accountability é descrita como um processo no qual uma pessoa

\footnotetext{
${ }^{1}$ CPS descreve sistemas como robôs, drones, veículos e sistemas de controle industrial, que usam sensores, software e atuadores para detectar, monitorar e controlar ou influenciar o mundo físico.
} 
tem a obrigação de explicar suas ações a outra parte que tem o direito de julgar essas ações e administrar possíveis consequências positivas ou negativas em resposta a elas.

Este trabalho parte da necessidade de investigar como accountability é associada a SI, em uma visão transversal e multidisciplinar de outras áreas de conhecimento com SI, com o objetivo de buscar soluções que estimulem a sustentabilidade ${ }^{2}$ de sistemas.

Esta premissa decorre da proposta de estudo de inserir mecanismos de accountability a sistemas, que passariam ser conhecidos como sistemas accountable. Para tanto defendemos que três fundamentos devem estar presentes em sistemas: engajamento, gerenciamento e observância a regras (compliance).

Pretendemos com esta pesquisa avançar em uma nova fronteira para tratar accountability em SI. Para alicerçar o embasamento, está sendo realizado um mapeamento sistemático da literatura cobrindo um conjunto de bases de conhecimentos que julgamos importantes para SI. Esta análise é primordial, pois visa fundamentar a construção de um framework para avaliação de accountability em SI, com a finalidade de fornecer informações relevantes extraídas de sistemas de forma a evidenciar a accountability, ajudando a demonstrar os impactos na sustentabilidade de sistemas.

\section{Apresentação do Problema}

SI estão se tornando cada vez mais complexos ${ }^{3}$, haja visto o crescimento de elementos que compõem os sistemas, as interações necessárias para seu funcionamento, a diversidade de tecnologias, o compartilhamento de dados, as demandas dos usuários, para além da limitação de equipamentos e plataformas [Maciel et al. 2016; Fernandes et al. 2018].

Desta forma, teorizar sobre accountability em SI é uma questão complexa, pois exige a construção de mecanismos preventivos e preditivos nas atividades dos sistemas para assegurar sua sustentabilidade. Tais atividades são compostas por múltiplas variáveis, além de interações diversas entre usuários e outros sistemas, o que sinaliza a emergência de se buscar mecanismos accountable que fortaleçam o engajamento nos usuários, a gerenciamento dos recursos e atividades e observância a regras (compliance), Figura 1.

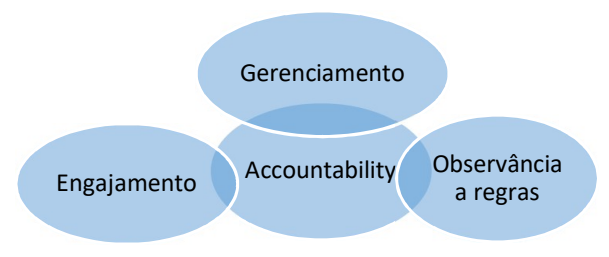

Figura 1 - Proposta de princípios de accountability para sistemas

Defendemos que o sucesso de um sistema está associado ao engajamento de seus usuários, uma vez que, a partir do senso de pertencimento, os sistemas tendem a se beneficiar do ciclo de feedbacks dos usuários, evidenciando potencialidades como: melhorias sistemáticas, sinalizações de erros, valorização da própria atividade (ou

\footnotetext{
${ }^{2}$ Sustentabilidade é a capacidade de perseverar e preservar a função de um sistema durante um período prolongado de tempo de efeitos sociais, técnicos e ambientais [Lago et al. 2015].

3 Sistema complexo é um sistema sociotécnico coletivo com muitos componentes independentes e interdependentes, que interagem de maneira não linear (o comportamento não pode ser expresso como somatórios da atividade de componentes individuais) e possuem interdependências que são difíceis de descrever, prever e projetar [Alampalli e Pardo 2014].
} 
trabalho), compartilhamento de objetivos locais ou globais entre todos, e assim substancialmente se manter sustentável.

Investigar essa adequação de SI com a primazia de accountability envolve mudanças nas culturas dos atores, principalmente aquelas que impactam negativamente a saúde geral do sistema e que tendem ser de difícil mensuração. Há necessidade de prever sintomas como culpa e desculpas para errar ou para justificar os erros. Identificar essas características contribui na estipulação de políticas de fomento ao engajamento que, por consequência, aumenta a colaboração e senso de pertencimento [Van Bussel 2016; Kacianka e Pretschner 2018].

Ao defendermos o engajamento como meta para alcançar o sucesso dos SI em ambientes complexos, os desenvolvedores devem refletir os contextos e avaliar se os sistemas estão entregando as soluções com base no que o gerenciamento determina. Para tantas atividades, recursos tecnológicos, plataformas, dados, demandas e especificidades, o gerenciamento precisa ser pensando em diferentes dimensões, considerando aspectos sociais, técnicos, ambientais e econômicos.

Ao sistematizar accountability em SI, estamos problematizando a exigência da avaliação dos mecanismos de gerenciamento eficientes em observância a sustentabilidade, pela busca da previsibilidade do que fazer, quando fazer e de que forma fazer para alcançar as metas dos sistemas com a menor quantidade de recursos (humanos ou tecnológicos) no menor intervalo de tempo, a partir dos princípios de qualidade de sistemas e produtos de softwares: eficácia, eficiência, satisfação, liberdade do risco e cobertura de contexto [ISO/IEC 25023 2016].

Mostra-se desafiadora a vinculação de iniciativas de gerenciamento que, associadas ao engajamento, possam contribuir para compartilhar objetivos. Segundo Araujo (2016), a mudança de paradigma só será alcançada quando os sistemas oferecerem mecanismos que estimulem atores e instituições a cooperar e buscar maneiras de: i) compartilhar e construir efetivamente objetivos comuns, ii) melhorar continuamente processos, iii) construir soluções que possam ajudar as pessoas a entenderem como a organização funciona e se comporta, e iv) ajudar indivíduos e organizações a produzir de maneira colaborativa e independente de crenças e hábitos, guiando a compreensão do senso de que atitudes individuais impactam todo o sistema.

Por outro lado, um processo de gerenciamento accountable necessita de políticas de regulação. A observância a regras em sistemas accountable devem ser transparentes a todos, ou seja, as regras e fundamentos que concretizam o controle exigem uma parametrização ordenada. Há que se criar um ambiente com um conjunto de políticas onde se mostre efetivamente o que é certo e o que é errado, cabendo inclusive graus de sanções. Contudo, o viés punitivo não é meramente uma ferramenta para instrumentalizar a culpa, mas sim um alicerce para evidenciar indicações de como manter o senso de pertencimento entre os envolvidos.

Logo, observância a regras assegura o acompanhamento do controle e engajamento, por meio de políticas e práticas, alicerçadas nos conceitos e demandas do sistema e sujeita a sanções quando há ausência de accountability. Um SI accountable deve deixar claro o que é esperado de uma funcionalidade ou comportamento dos atores e de sistemas, bem como sinalizar os impactos, consequências e sanções.

Este tripé de fundamentos de accountability em SI evidencia o entendimento e correlação em ciclos de causa e efeito em sistemas complexos, nos quais pelo coletivo se criam padrões, as informações emergem e, em alguns casos, se evolui e se aprende com os processos [Mitchell 2009]. 
Observa-se pelo exposto que correlacionar práticas, hábitos e recursos associados ao rastreamento das ocorrências de accountability em SI não é trivial. Mesmo a teoria de accountability do IS.Theorizeit.org apresenta uma teorização de forma discreta sem, por exemplo, proporcionar discussão ao tema.

Nesta pesquisa, buscamos fortalecer a discussão de accountability na área de SI, contribuindo com a criação de um framework conceitual e com uma investigação para avançar na teorização sobre o tema.

Para isso, pretendemos realizar a inclusão de mecanismos de aperfeiçoamento do engajamento, gerenciamento e observância a regras como estratégia para estimular resultados positivos e melhorar a sustentabilidade dos sistemas, na medida que serão aperfeiçoadas as funcionalidades e responsabilização dos envolvidos.

\section{Proposta de Solução}

Pretendemos com este estudo elaborar um framework conceitual de accountability em SI com base no relatório ISO/IEC 42030 (2013), que normatiza a elaboração de arquiteturas para frameworks, para auxiliar desenvolvedores, atores e pesquisadores a entenderem melhor os impactos da accountabillity em sistemas complexos, de formar a evidenciar como determinadas ações relacionadas a ciclos de causa e efeito impactam na sustentabilidade em sistema.

O objetivo da pesquisa é evidenciar as condições dos SI mediante os três princípios defendidos nesta estudo e os impactos na sustentabilidade de sistemas, a partir das dimensões de aspectos sociais, ambiental, técnicos e econômicos [Lago et al. 2015].

A dimensão econômica se preocupa com a preservação dos recursos. A dimensão social está preocupada com manutenção das relações. A dimensão ambiental busca melhorar o bem-estar humano e proteção dos recursos naturais. Por fim, a dimensão técnica se preocupa em apoiar o uso a longo prazo e a evolução dessa utilização intensiva dos sistemas. Desta forma, o diagnóstico da accountability e as entradas/saídas geradas por atores/sistemas - discriminados em dimensões de sustentabilidade - tendem a melhorar os processos de tomada de decisão, potenciais intervenções e sanções.

Além da construção do framework para ajudar profissionais a explorar os sistemas com abordagem accountable, esta pesquisa pretende contribuir com a teorização a respeito do tema para área de SI, fornecendo discussões para teorização no portal de teorias de $\mathrm{SI}^{4}$ com base na técnica de mapeamento sistemático da literatura.

Este mapeamento está sendo conduzido nas bases de dados da ACM Digital Library, IEEE Xplore, Scopus, Science Direct, Engineering Village e SpringerLink, visando buscar evidências do estado da arte a partir da seguinte questão de pesquisa: "Como accountability é definida no contexto de SI?"

\section{Projeto de Avaliação da Solução}

O framework será aplicado em dois potenciais sistemas complexos: i) um colégio de aplicação formado por um conjunto de unidades escolares da rede pública do Estado do Rio de Janeiro, com diferentes faixas etárias que dividem recursos e compartilham o mesmo espaço físico; e ii) um hospital da rede particular com atendimento a pacientes com câncer que utilizam artefatos formais de sistemas, tais como portais de periódicos e prontuários médicos para diagnósticos de pacientes, combinados a artefatos informais,

\footnotetext{
${ }^{4}$ Accountability theory - https://is.theorizeit.org/wiki/Accountability_theory
} 
como serviços de troca de mensagens entre médicos, evidenciando a complexidade das barreiras neste ecossistema.

Os dados serão coletados mediante avaliação do perfil dos usuários, identificação do ciclo de culpabilidade como forma de demonstrar o nível de accountability individual, além de entrevistas com usuários estratégicos dos sistemas para criar os contextos para avaliação do framework. Além disso, está previsto um levantamento tecnologias existentes e os sistemas que interoperam, com objetivo de criar um conjunto de informações que demonstrem o estado da sustentabilidade do sistema.

\section{Atividades Realizadas}

Para compreensão do tema em área de conhecimento diversa à SI, foi realizado previamente um mapeamento sistemático da literatura acerca de soluções e abordagens de accountability na área de Educação. Esta área foi escolhida por apresentar diferentes níveis de sistemas, desde sistemas locais relacionados ao funcionamento do cotidiano escolar a sistemas de governos na forma de portais.

Foi realizado também um levantamento nas bases de patentes do Instituto Nacional da Propriedade Industrial (INPI - nacional) e do United States Patent and Trademark Office (USPTO - americana). Este estudo buscou por evidências do estado da prática, onde a essência do trabalho consistia em identificar artefatos tecnológicos associáveis a accountability. Este tipo de pesquisa é importante por agregar resultados de outras abordagens de pesquisa, complementando o estado da arte, apresentado no ICEIS/SLIS 2018 [Cordeiro e Araujo 2018].

Ambas análises serviram para evidenciar a complexidade do ambiente educacional, o que gerou uma investigação da técnica de System Thinking ${ }^{5}$ como recurso para mapear accountability em Systems-of-Systems, trabalho que será apresentado no IEEE/ACM ICSE SESoS/WDES 2019 [Cordeiro e Santos 2019].

\section{Considerações Finais}

Neste trabalho, elencamos a necessidade de uma agenda de accountability em SI, almejando um olhar mais amplo para teorização de accountability, por meio da investigação de como a área de SI trata o tema e como este se apresenta nos SI em outras áreas de conhecimento. Desejamos avançar em uma investigação transversal e multidisciplinar, alicerçada no aperfeiçoamento dos sistemas em prol da melhor sustentabilidade em SI. Para alcançar tais objetivos, pretendemos desenvolver um framework que analisa a accountability em SI com base no relatório ISO/IEC 42030 (2013) que propõe modelagem para arquiteturas e em estudos experimentais de literatura.

\section{Referências}

Alampalli, S., Pardo, T., 2014. A study of complex systems developed through public private partnerships, in: Proceedings of the 8th International Conference on Theory and Practice of Electronic Governance. ACM, pp. 442-445.

Araujo, R., 2016. Information Systems and the Open World Challenges. I GranDSI-BR 42-51.

\footnotetext{
${ }^{5}$ System Thinking é a base de uma metodologia para o pensamento crítico, onde um sistema complexo é analisado em termos de seus muitos componentes, variáveis, pessoas, recursos etc. A técnica busca diminuir os limites dos sistemas, para melhor defini-los, especialmente em termos de interações.
} 
Cordeiro, F., Araujo, R., 2018. Tecnologias no Cotidiano Escolar: Levantamento de Patentes no Brasil e Estados Unidos de 2000 a 2017, in: XLIV Conferência Latino-Americana de Informática. Presented at the SLIS - Simpósio Latinoamericano em Informática e Sociedade.

Cordeiro, F., Santos, R., 2019. Systems Thinking as a Resource for upporting Accountability in System-of-Information-Systems: Exploring a Brazilian School Case, in: International Conference on Software Engineering. Presented at the SESoS/WDES, ICSE 2019, Montréal, Canada.

de Wolf, I.F., Janssens, F.J., 2007. Effects and side effects of inspections and accountability in education: an overview of empirical studies. Oxford Review of education 33, 379-396.

Feigenbaum, J., Jaggard, A.D., Wright, R.N., Xiao, H., 2012. Systematizing "accountability" in computer science (version of feb. 17, 2012). Technical Report YALEU/DCS/TR-1452, Yale University, New Haven, CT.

Fernandes, J.C., Neto, V.V.G., Santos, R.P. dos, 2018. Interoperability in Systems-ofInformation Systems: A Systematic Mapping Study, in: Proceedings of the 17th Brazilian Symposium on Software Quality. ACM, pp. 131-140.

ISO/IEC 25023, 2016. Systems and software Quality Requirements and Evaluation (SQuaRE) - System and software quality models. International Organization for Standardization.

ISO/IEC/IEEE FDIS 42030, 2013. Software, systems and enterprise -- Architecture evaluation framework. International Organization for Standardization.

Kacianka, S., Pretschner, A., 2018. Understanding and Formalizing Accountability for Cyber-Physical Systems, in: 2018 IEEE International Conference on Systems, Man, and Cybernetics (SMC). pp. 3165-3170.

Lago, P., Koçak, S.A., Crnkovic, I., Penzenstadler, B., 2015. Framing sustainability as a property of software quality. Communications of the ACM 58, 70-78.

Maciel, R.S.P., David, J.M.N., Claro, D.B., Braga, R., 2016. Full Interoperability: Challenges and Opportunities for Future Information Systems. I GranDSI-BR 107-118.

Medeiros, A.K., Crantschaninov, T.I., da Silva, F.C., 2013. Estudos sobre accountability no Brasil: meta-análise de periódicos brasileiros das áreas de administração, administração pública, ciência política e ciências sociais $47,745-775$.

Mitchell, M., 2009. Complexity: A guided tour. Oxford University Press.

Mulgan, R., 2000. 'Accountability': An ever-expanding concept? Public administration $78,555-573$.

Van Bussel, G.-J., 2016. An accountability challenge: Capturing records and their context in enterprise information systems, in: 10th European Conference on Information Systems Management, ECISM 2016, September 8, 2016 - September 9, 2016, Proceedings of the European Conference on IS Management and Evaluation, ECIME. Academic Conferences and Publishing International Limited, pp. 204211. 